\title{
CONOCIMIENTO QUE TIENEN SOBRE EL CLMATERIO MUJ ERES ENTRE 40-64 AÑOS USUARIAS DE ATENCIÓN PRIMARIA
}

\author{
Claudia Gómez M. ${ }^{a}$, Carolina Carmi K. ${ }^{a}$, Victoria Espinosa F. PhD ${ }^{1 b}$, Silvia Sepúlveda B. \\ $P h D^{1 c}$, Fanny López A. PhD'a \\ ${ }^{1}$ Escuela de Medicina, Laboratorio de Investigación Científica Emory Black, Facultad de Ciencias Médicas, Universidad

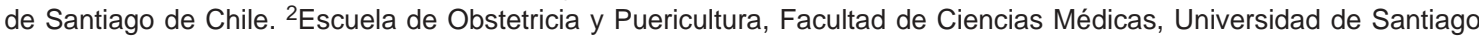 \\ de Chile.
}

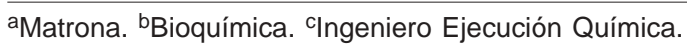

\section{RESUMEN}

Objetivo: Determinar el grado de conocimiento de la fase del climaterio y su relación con el nivel de instrucción que tiene un grupo de mujeres de 40-64 años usuarias de atención primaria. Método: La muestra de tipo probabilística compuesta por 150 mujeres de 40 a 64 años, a quienes se aplicó una encuesta validada de preguntas de alternativas múltiples, puntuadas para su evaluación. Las variables estudiadas fueron divididas en tres grupos: a) aquellas que definen el perfil sociodemográfico de las mujeres; b) variables relacionadas con el grado de conocimientos de causas, síntomas, tratamientos, cuidados, cambios en la salud y calidad de vida de la etapa climatérica; c) fuentes de información del conocimiento. El análisis de estas variables se realizó a través de la determinación de medidas de tendencia central y la prueba de Kruskal-Wallis, con un $\mathrm{p}<0,05$. Resultados: El perfil de las mujeres en este estudio fue: edad promedio $51,89 \pm 7,35$ años, $52 \%$ casadas, $52 \%$ previsión FONASA-A (pobreza e indigencia) y $60 \%$ con educación media incompleta. Solo el $11 \%$ de la población encuestada refirió haber asistido a talleres del tema, y un $40 \%$ refirió haber recibido información de médicos y/o matronas. En relación al conocimiento general de la población sobre la etapa climatérica, un $72 \%$ tiene un nivel medio y un $2 \%$ nivel bajo de conocimiento, respectivamente. Conclusiones: No existe relación estadísticamente significativa entre el grado de conocimiento sobre la etapa climatérica y la edad de las mujeres en el estudio, así como tampoco por la adquisición de información de fuentes válidas, pero sí con el nivel educacional de las mismas.

\section{PALABRAS CLAVES: Climaterio, conocimiento, menopausia}

\section{SUMMARY}

Objective: To determine the grade of knowledge of the climateric phase and their relationship with the instruction level that has a group of 40-64 year-old women users of primary care. Method: The sample was probabilistics made up of 150 women of 40 to 64 years and were submitted to a validated survey of questions with multiple answers, punctuated for its evaluation. The variables included in this study were divided in three groups: a) those that define the social-demographic profile of the women; b) variables related with the grade of knowledge of causes, symptoms, treatments, cares, changes in the health and quality of life of the climateric stage; c) sources of information. The analysis of these variables was carried out through the determination of measures of central tendency, and the non parametric Kruskal-Wallis test 
with a significance level $p<0.05$. Results: The profile of the women studied was, $51.89 \pm 7.35$ years, $52 \%$ married, $52 \%$ health forecast FONASA-A (poverty and indigence) and $60 \%$ with incomplete high school. Only the $11 \%$ of the interviewed population referred to have attended lectures of the topic, and $40 \%$ refers to have received doctors' information and/or midwife. In relation to the population's general knowledge on the climateric stage, $72 \%$ has a medium level of knowledge and $2 \%$ had low level. Conclusions: Did not exists relationship with significant statistics among the grade of knowledge on the climateric stage and age of the women in the study, as well as neither for the acquisition of information from valid sources but exists with the educational level of the women.

\section{KEY WORDS: Climacterium, knowledge, menopause}

\section{INTRODUCCIÓN}

Los cambios biodemográficos de la población chilena, caracterizados por una mayor expectativa de vida, han producido un incremento en la frecuencia de mujeres que alcanza la etapa de climaterio $(1,2)$. Así lo comprueba el Censo del 2002, señalando que a nivel nacional, el número de mujeres de 40 a 64 años es de 2.007.320 y a nivel de la Región Metropolitana de 836.449 mujeres en periodo perimenopáusico (3).

El climaterio o perimenopausia se refiere al periodo previo, concomitante y posterior a la menopausia, en el cual se establecen los cambios endocrinos, biológicos y clínicos (4). Se caracteriza por la transición de la vida reproductiva a la no reproductiva de la mujer (5). Su duración es variable, pero por lo general, se considera desde la declinación de la función ovárica en una mujer de más de 40 años, hasta que deja de tener periodos menstruales durante un año, siendo éste el síntoma más importante de esta transición $(6,7)$.

Respecto del nivel de conocimientos estudiados, podemos referir que una población de mujeres premenopáusicas chinas demostró poseer un mejor nivel de conocimiento respecto de síntomas y tratamientos hormonales de la menopausia que mujeres en etapa postmenopáusica (8). De igual manera un estudio realizado en una población de mujeres andaluzas, describe que la experiencia de la menopausia, o la utilización de los servicios sanitarios en relación a ésta, no parece influir en el conocimiento sobre la etapa climatérica y sus cuidados (9). Por el contrario, un grupo de investigadores cubanos refiere que su población en climaterio, posee un adecuado nivel de conocimiento respecto del tema, debido a la política de salud nacional permite que los recursos de salud estén al alcance de toda la población, que tiene un alto nivel de escolaridad y recibe educación para la salud a través de los medios de difusión masiva (10). En un estudio realizado en Chile en 1994, con 494 usuarias de consulta hospitalaria,
$93,5 \%$ de ellas opinaba que la menopausia se acompañaba de efectos adversos sobre la salud y un porcentaje que no supera el $60 \%$, conocía los beneficios de la terapia de reposición hormonal (TRH) y tenía alguna noción sobre los efectos secundarios de la misma (11). Las 3 fuentes principales de información de estas mujeres, fueron los medios de difusión masiva (televisión, radio, revistas), los amigos-conocidos y finalmente el médico; un elevado porcentaje de estas mujeres se consideraron insuficientemente informadas acerca del climaterio y su tratamiento (11).

La atención de la mujer centrada en un modelo holístico, y basado en el concepto de salud de la OMS, se caracteriza por una visión amplia, biológica y psicosocial, de los procesos que ocurren durante la transición climatérica. Por ende, es imprescindible conocer, cual es la información que sobre este tema, tienen las mujeres que se encuentran en esta etapa, para poder entregar una atención integral de calidad y enfocada a superar, especialmente las debilidades que se detecten en las mujeres climatéricas (12).

El objetivo de este estudio es determinar el grado de conocimiento de la fase del climaterio y su relación con el nivel de instrucción que tiene un grupo de mujeres de 40-64 años, usuarias del Centro de Salud №5 de la comuna de Santiago, Región Metropolitana, Chile.

\section{PACIENTES Y MÉTODO}

La muestra fue seleccionada de un total de mujeres entre 40 y 64 años usuarias del Centro de Salud №5 de la comuna de Santiago, realizada en los meses de julio y agosto del 2006. La muestra fue de tipo no probabilística y dividida en 30 mujeres por quinquenio etáreo, totalizando una muestra de 150 mujeres. El criterio de inclusión fue: mujeres entre 40 y 64 años con estado de salud normal (definido por el National Center for Health Statistics, como aquella condición compatible con el desempeño de las actividades rutina- 
rias). El criterio de exclusión fue: mujeres con patologías psiquiátricas o de déficit mental que pudiera dificultar la interpretación de las preguntas.

Las variables se clasificaron en: a) variables que definen el perfil sociodemográfico de las participantes (edad, nivel educacional, estado civil, tipo de previsión de salud); b) variables relacionadas con el grado de conocimiento de causas y síntomas de la etapa climatérica, cambios en la salud y calidad de vida y por último conocimientos sobre tratamientos y cuidados en el climaterio; c) fuentes de información del conocimiento adquirido sobre el climaterio. A partir de esta categorización de variables se construyó una escala de puntajes para cada ítem de conocimientos que se describe en Tabla I.

El perfil social de las pacientes, en lo relativo al tipo de previsión en salud, corresponden mayoritariamente al sistema público del Fondo Nacional de Salud (FONASA) y privado de las Instituciones de Salud Previsional (ISAPRES). Los beneficiarios del FONASA se dividen en las siguientes 4 categorías: FONASA A: carentes de recursos 0 indigentes; FONASA B: ingreso imponible mensual menor o igual a $\$ 144.000$; FONASA C: ingreso imponible mensual mayor a $\$ 144.000$ y menor o igual a \$210.240; FONASA D: ingreso imponible mensual mayor a $\$ 210.240$ (equivalencia monetaria: 1 dólar americano=522,99 pesos chilenos, promedio al 31 de agosto de 2007).

La recolección de datos para este estudio se realizó mediante una encuesta elaborada por las autoras, la cual consta de preguntas relacionadas con el grado de conocimiento sobre las causas, manifestaciones y tratamientos propios del período de climaterio. Dicho cuestionario consiste en 17 preguntas de alternativas múltiples, a las que se asignó un puntaje (puntaje total: 0 y 39 puntos).

El instrumento fue validado a través de la revisión de sus contenidos por profesionales del área y por la aplicación de la prueba piloto en un $10 \%$ de la muestra (16 mujeres entre 40 y 64 años de la comuna de Quinta Normal, Región Metropolitana).

Se determinó la confiabilidad del instrumento mediante el coeficiente de relación pre y post test, donde no hubo diferencia significativa entre las 2 pruebas.

Se solicito a las mujeres participantes del estudio su consentimiento informado y escrito.

La tabulación de los datos se realizó en planillas de Microsoft Excel 2003 y su cuantificación, en base a escalas de puntuaciones asignadas por ítem de acuerdo a lo descrito en la definición de variables. Para el análisis estadístico se utilizó la determinación de medidas de tendencia central y la prueba no paramétrica de Kruskal-Wallis (programa estadístico Graphpad Prism 4.02), con un nivel de significación estadística de $p<0,05$.

\section{RESULTADOS}

Las mujeres incorporadas a este estudio, tienen una edad promedio de 51,89 $\pm 7,35$ años, casadas $(52 \%)$, que utilizan el sistema de salud dirigido a la población de escasos recursos (FONASA-A 52\%) y con un nivel de instrucción cercano al $60 \%$ de la población sin enseñanza media completa (Tabla II).

Los conocimientos sobre la etapa climatérica, en el $11 \%$ de la población encuestada fueron adquiridos por asistencia a talleres relacionados con este tema, mientras que la fuente de información

Tabla I

DEFINICIÓN DE RANGOS DE PUNTAJE ASIGNADO A VARIABLES ASOCIADAS A GRADO DE CONOCIMIENTO

\begin{tabular}{lll}
\hline Variable & Subvariable & Definición de los Puntajes \\
\hline & Sobre causas y síntomas de la etapa climatérica & Alto (13 a 17 puntos) \\
& & Medio ( 7 a 12 puntos $)$ \\
Nivel de conocimiento & Bajo ( $\leq$ a 6 puntos) \\
& Sobre cambios en la salud y la calidad de vida & Alto (10 a 14 puntos) \\
& Medio (5 a 9 puntos) \\
& Bajo ( $\leq$ a 4 puntos) \\
& Sobre tratamientos y cuidados en el climaterio & Alto ( 6 a 8 puntos $)$ \\
& Medio (3 a 5 puntos) \\
& Bajo ( $\leq$ a 2 puntos)
\end{tabular}

Puntajes: Alto equivale a 28 a 39 puntos; Medio equivale a 15 a 27 puntos; Bajo equivale $\leq$ a 14 puntos. 
Tabla II

DISTRIBUCIÓN DEL PERFIL SOCIODEMOGRÁFICO DE LAS MUJERES PERIMENOPAUSICAS ESTUDIADAS

\begin{tabular}{|c|c|c|c|c|c|c|}
\hline Edad promedio & \multicolumn{2}{|c|}{ Nivel educacional } & \multicolumn{2}{|c|}{ Estado civil } & Previsión & $\%$ \\
\hline \multirow{7}{*}{$51,89 \pm 7,35$} & Básica incompleta & 25,3 & Casada & 52,0 & Fonasa $\mathrm{A}$ & 52,0 \\
\hline & Básica completa & 9,3 & Soltera & 14,7 & Fonasa B & 24,0 \\
\hline & Media incompleta & 26,7 & Conviviente & 10,7 & Fonasa C & 12,0 \\
\hline & Media completa & 20,0 & Viuda & 8,0 & Fonasa D & 8,0 \\
\hline & Superior incompleta & 5,3 & Separada & 14,7 & Isapre & 2,7 \\
\hline & Superior completa & 13,3 & & & Otros & 1,3 \\
\hline & Ninguna & 0,0 & & & & \\
\hline
\end{tabular}

válida más frecuentemente citada correspondió a matrona (24\%) (Tabla III).

Los tópicos relacionados con el climaterio fueron abordados a través de preguntas de respuesta múltiple. Cuando se preguntó respecto de la causa del climaterio, las mujeres encuestadas respondieron acertadamente en un $85,3 \%$, señalando la disminución de las hormonas en su organismo y fueron capaces de reconocer la sintomatología propia de este período, indicando con mayor frecuencia los cambios de ánimo (94,7\%), la irritabilidad $(80 \%)$, los bochornos (92\%), las sudoraciones $(75,3 \%)$, los dolores óseos y cabeza (69\% aproximadamente en ambos). Por el contrario ellas asociaron erróneamente, el climaterio con la halitosis en un $42,6 \%$ de las oportunidades. Respecto de cambios en la salud y calidad de vida, la gran mayoría de las mujeres (72\%) reconocen la disminución de la lubricación vaginal, la disminución de la libido $(54,7 \%)$ y la dispareunia $(68,6 \%)$, pero erróneamente, no asocian este período con un aumento de la frecuencia de infecciones urinarias $(60 \%)$. De las patologías que la población asocia al climaterio, en un $80 \%$ refieren osteoporosis, en un porcentaje mucho menor indican problemas cardiovasculares (25\%) y cabe señalar, que reconocen como factor de riesgo de cáncer de mama asociado con antecedentes maternos en un $76 \%$ y un $61,3 \%$ al uso de terapia de reemplazo hormonal. Al abordar el tema de tratamientos y cuidados durante el climaterio, un gran porcentaje de la población (79\%) conoce la existencia de algún tratamiento para atenuar las molestias propias de esta etapa y de este grupo de mujeres, casi en su totalidad mencionan la terapia de reemplazo hormonal (96\%). Respecto de las medidas no farmacológicas para atenuar los síntomas del climaterio, todas ellas (evitar alcohol, tabaco, café, control de
Tabla III

DISTRIBUCIÓN DE LAS FUENTES DE INFORMACIÓN SOBRE CLIMATERIO QUE REFIEREN LAS MUJERES PERIMENOPAUSICAS ESTUDIADAS

\begin{tabular}{lrr}
\hline Fuente de información sobre climaterio & $n$ & $\%$ \\
\hline Matrona & 36 & 24,0 \\
Médico & 24 & 16,0 \\
Amiga & 22 & 14,7 \\
Vecina & 6 & 4,0 \\
Familiar & 4 & 2,7 \\
Revistas, diario & 13 & 8,7 \\
Televisión & 8 & 5,3 \\
Otro & 37 & 24,7 \\
& & \\
\hline Total & 150 & 100,0 \\
\hline
\end{tabular}

niveles de colesterol, ejercicio físico, y dieta equilibrada) fueron acertadamente referidas por más de un $75 \%$ de la población, pero dentro de la alimentación saludable sólo un $30 \%$ de la población reconoce la necesidad de una dieta baja en sodio. Por el contrario la exposición moderada a la luz solar fue considerada innecesaria por más del $46 \%$ de las encuestadas.

Al evaluar la encuesta según puntajes, se identificaron grados de conocimiento uniformes en las distintas variables estudiadas, determinando que las mujeres encuestadas poseían un mediano grado de conocimiento respecto a las causas y sintomatología de la etapa climatérica (51\%), sin embargo, poseen un mediano grado de conocimiento en relación a los cambios en la salud y calidad de vida (75\%), y tratamientos y cuidados (53\%) existentes en dicha etapa, lo que derivó que al analizar la encuesta en su conjunto se obtuviera que un $72 \%$ de la población posee un grado medio de conocimiento (Tabla IV). 
Tabla IV

\section{DISTRIBUCIÓN DEL NIVEL DE CONOCIMIENTOS SOBRE CLIMATERIO QUE TIENEN LAS MUJERES PERIMENOPAUSICAS ESTUDIADAS}

\begin{tabular}{|c|c|c|c|}
\hline Tema en que se evalúa conocimiento & Nivel de conocimiento & $n$ & $\%$ \\
\hline \multirow{3}{*}{ Causas y síntomas de la etapa climatérica } & Alto & 69 & 46 \\
\hline & Medio & 77 & 51 \\
\hline & Bajo & 4 & 3 \\
\hline \multirow{5}{*}{ Cambios en la salud y la calidad de vida } & Total & 150 & 100 \\
\hline & Alto & 28 & 19 \\
\hline & Medio & 113 & 75 \\
\hline & Bajo & 9 & 6 \\
\hline & Total & 150 & 100 \\
\hline \multirow{3}{*}{ Tratamientos y cuidados en el climaterio } & Alto & 45 & 30 \\
\hline & Medio & 80 & 53 \\
\hline & Bajo & 25 & 17 \\
\hline \multirow{5}{*}{ Conocimiento general de la etapa climatérica } & Total & 150 & 100 \\
\hline & Alto & 39 & 26 \\
\hline & Medio & 108 & 72 \\
\hline & Вајо & 3 & 2 \\
\hline & Total & 150 & 100 \\
\hline
\end{tabular}

Al relacionar el nivel educacional con el grado de conocimiento que las encuestadas referían de cada tópico en forma independiente (causas, síntomas del climaterio, cambios de salud y calidad de vida en esta etapa, tratamientos y cuidados), no encontramos relación entre cada tópico y el nivel educacional. Pero al evaluar la encuesta en su totalidad contemplando los 3 aspectos en conjunto, si se verificó relación (Tabla V), observándose que existió diferencia estadísticamente significativa, analizada a través del parámetro $\mathrm{H}$ de Kruskal-Wallis $(p=0,0365)$, entre el nivel de conocimiento y el nivel de instrucción de la población.

Al realizar similar análisis relacionando la edad de las encuestadas clasificadas por quinquenios, en relación al conocimiento de esta etapa, no existió diferencia significativa entre los diferentes grupos etéreos (Tabla $\mathrm{VI}$ ).

Por último cuando se analizo la influencia de la adquisición de información desde fuentes válidas (entendiéndose por válida aquella referida por profesionales de la salud médicos y/o matronas) con el nivel de conocimiento exhibido por nuestras encuestadas (Tabla VII), se observó que la diferencia entre los grupos no fue estadísticamente significativa, evaluada a través del parámetro $U$ de Mann-Whitney.

\section{DISCUSIÓN}

Dentro del perfil sociodemográfico, se destaca que no hubo analfabetas y las categorías predominantes fueron enseñanza básica incompleta $(25,56 \%)$ y enseñanza media incompleta $(26,67 \%)$. Esto es un punto destacable, a diferencia de un estudio realizado en Perú sobre el nivel de conocimiento y aceptación de la terapia de reemplazo hormonal en mujeres mayores de 39 años, donde

\section{Tabla V}

RELACIÓN ENTRE EL NIVEL EDUCACIONAL Y EL GRADO DE CONOCIMIENTO SOBRE CLIMATERIO QUE PRESENTAN LAS MUJERES PERIMENOPAUSICAS ESTUDIADAS

\begin{tabular}{lrrrr}
\hline Nivel de educación & \multicolumn{4}{c}{ Grado de conocimiento } \\
Grupos & Alto & Medio & Bajo & Total \\
\hline Básica incompleta & 9 & 28 & 1 & 38 \\
Básica completa & 5 & 9 & 0 & 14 \\
Media incompleta & 14 & 25 & 1 & 40 \\
Media completa & 4 & 26 & 0 & 30 \\
Superior incompleta & 0 & 8 & 0 & 8 \\
Superior completa & 8 & 12 & 0 & 20 \\
\hline Total & 40 & 108 & 2 & 150 \\
\hline
\end{tabular}

$p=0,0365$. 
Tabla VI

\section{RELACIÓN ENTRE GRUPO ETÁREO Y EL GRADO DE CONOCIMIENTO SOBRE CLIMATERIO QUE TIENEN LAS MUJERES PERIMENOPAUSICAS ESTUDIADAS}

\begin{tabular}{lcccc}
\hline \multirow{2}{*}{$\begin{array}{l}\text { Grupo etáreo } \\
\text { Años }\end{array}$} & \multicolumn{4}{c}{ Grado de conocimiento } \\
& Alto & Medio & Bajo & Total \\
\hline $40-44$ & 10 & 19 & 1 & 30 \\
$45-49$ & 10 & 20 & 0 & 30 \\
$50-54$ & 8 & 21 & 1 & 30 \\
$55-59$ & 8 & 22 & 0 & 30 \\
$60-64$ & 5 & 25 & 0 & 30 \\
\hline Total & 41 & 107 & 2 & 150 \\
\hline
\end{tabular}

$p=0,6665$.

se encontró un $12,7 \%$ de analfabetas y la categoría más predominante fue la educación básica $(50 \%)(13)$. Este factor fue importante para nuestra investigación, pues todas las encuestadas presentaron algún nivel de escolaridad, lo que según estudios anteriores facilita el entendimiento y la comprensión de la encuesta y la futura educación sobre el climaterio (13).

De acuerdo con nuestro estudio, fueron muy pocas las mujeres asistentes a talleres relacionados con el climaterio, en algún momento de su vida $(11 \%)$. Esto podría reflejar, el poco interés por parte de las mujeres en conocer más sobre este tema o el escaso acceso a programas educativos. También se encuentra una falta de educación individual en las atenciones, pues según los resultados del estudio se describe que, sólo un $40 \%$ de las mujeres recibieron información sobre el climaterio de parte de profesionales de la salud (médico y matrona). Resultados parecidos se encontraron en un estudio realizado en Perú sobre conocimiento y actitudes de la mujer peruana sobre climaterio y menopausia, donde los principales medios de información sobre este tema, fueron la televisión y las revistas (14). Todas estas situaciones demuestran que las mujeres en edad climatérica deben ser educadas durante esta etapa y que esta entrega de conocimientos debe ser dinámica en los contenidos (14).

Respecto de las causas y sintomatología de la etapa climatérica, podemos señalar que aún existe en las mujeres una falta de comprensión en el tema, observación que coincide con otros autores $(4,15)$, por ejemplo, no reconocen algunos síntomas prevalentes de este período como es la ansiedad, sino que lo relacionan con el estrés de la vida diaria, como ha sido descrito anteriormente
Tabla VII

RELACIÓN ENTRE LA ADQUISICIÓN DE INFORMACIÓN DESDE FUENTES VÁLIDAS Y EL GRADO DE CONOCIMIENTO, SOBRE LA ETAPA CLIMATÉRICA, QUE PRESENTAN LAS MUJERES ESTUDIADAS

\begin{tabular}{lcccc}
\hline & \multicolumn{4}{c}{ Grado de conocimiento } \\
Recibe información & Alto & Medio & Bajo & Total \\
\hline $\mathrm{Si}$ & 20 & 48 & 1 & 69 \\
$\mathrm{No}$ & 19 & 60 & 2 & 81 \\
\hline Total & 39 & 108 & 3 & 150 \\
\hline
\end{tabular}

$p=0,6740$.

(15) y presentan una tendencia a vincular otros síntomas como la halitosis con el climaterio. Esto último refleja, una tendencia a vincular todas las manifestaciones negativas del cuerpo con la etapa climatérica (6).

Cuando consideramos los tópicos de cambios en la salud y calidad de vida durante el climaterio, llama la atención que un $60 \%$ de la población no reconoce una relación relevante, que es infecciones urinarias y climaterio (4). El resto de los cambios genitales que pueden producir variaciones de ánimo y de la autoestima, y conllevar a problemas en el desarrollo normal de su sexualidad, son reconocidos por la población estudiada (14).

Dentro de los problemas que nuestra población reconoce como asociada al climaterio se cuentan las patologías con sintomatología ósea y el cáncer de mama, esto coincide con resultados previamente publicados en una población mexicana (16). De igual manera en este estudio se señala el escaso conocimiento que presenta la población del aumento del riesgo de enfermedades cardiovasculares asociadas a la etapa climatérica. En nuestro país las consultas médicas por problemas óseos y ginecológicos asociadas al cáncer de mamas, son origen de consultas recurrentes, hecho que se reproduce en otras poblaciones (17).

Del conocimiento de tratamientos y cuidados en el climaterio se puede señalar que la mayoría (78\%) de las encuestadas reconoce algún tratamiento para la sintomatología de la etapa climatérica, siendo el principal la THR, hallazgo similar al de un estudio realizado en Perú sobre conocimiento y aceptación de esta terapia, donde el porcentaje de mujeres mayores de 39 años que sabían de la existencia de tratamiento para el climaterio fue de un $72 \%$ (13). 
La mayoría las encuestadas reconocen la importancia de un estilo de vida saludable incluyendo dentro de este tópico la disminución del consumo de alcohol, tabaco, café y el control periódico de los niveles de colesterol, junto con dieta equilibrada y ejercicio moderado. Datos similares a los obtenidos en el presente estudio, se han publicado con anterioridad, en una población perimenopáusica peruana, la mayoría de esas mujeres piensa que son importantes los ejercicios físicos, y la disminución del consumo de grasa y tabaco $(18,19)$.

En nuestro estudio determinamos que existía relación estadísticamente significativa entre el nivel de educación y nivel de conocimiento sobre el climaterio, lo que concuerda con estudios realizados en una población de mujeres españolas (20) y taiwanesas (21).

Al relacionar los grupos etáreos con el grado de conocimiento de las temáticas mencionadas sobre el climaterio, no se encontró relación entre estas variables. Esto llama la atención, ya que las mujeres de mayor edad deberían saber más sobre el tema, ya que han vivenciado más años esta etapa y también han tenido más tiempo para instruirse sobre el tema.

Al comparar la relación existente entre la adquisición de información desde fuentes válidas (médico y matrona) y la asistencia a talleres, con el grado de conocimiento de las temáticas en cuestión encontramos que no existe relación entre estas variables. Pero que al igual que en nuestro estudio, se detectó en una población perimenopáusica española, que la asistencia a centros de atención primaria y el nivel de conocimientos de la etapa climatérica, estaban influenciados por la edad y el nivel de instrucción de las pacientes (22).

\section{CONCLUSIÓN}

Nuestros resultados apuntan a demostrar que la población en estudio tiene un nivel de conocimientos medio del climaterio y que existe relación significativa con el nivel de instrucción, pero no con la edad, ni con el hecho de haber obtenido información de fuentes válidas.

\section{BIBLIOGRAFÍA}

1. Arriagada M, Arteaga E, Bianchi M, Brantes S, Montaño R, Osorio E, et al. Recomendaciones de tratamientos en la menopausia. ReV CHIL OBSTET GINECOL 2005;70:340-5.

2. Gramegna G, Blumel JE, Roncagliolo ME, Aracena
B, Tacla X. Patrones de conducta sexual en mujeres chilenas. Rev Med Chil 1998;126:162-8.

3. Comisión Nacional del XVII Censo de Población y VI de Vivienda. "Población total, por área urbanarural y sexo, según división político administrativa, grupos de edad y años de edad". Instituto Nacional de Estadísticas. Síntesis de resultados Censo 2002, Chile.

4. Arteaga E, Bianchi M. Fisiología del climaterio y menopausia. En: Pérez, A. Ginecología. 3aㅗ edición, Mediterráneo. Santiago de Chile. 2003. pp 179-85.

5. Garcia-Viniegras V, Regina C, Porta M. Climaterio y bienestar psicológico. Rev Cub Obstet Ginecol 2003;29(3):0-0. Disponible en: http://scielo.sld.cu/ scielo.php?script=sci_arttext\&pid=S0138$600 X 2003000300010 \&$ Ing=es\&nrm=iso\&tlng=es

6. Hurd W. Menopausia en Ginecología de Novak. 13 Ed. México. McGraw-Hill Interamericana. 2002. pp 891-914.

7. Prado C, Carmenante M, Martínez A. La terapia hormonal de reemplazo. ¿Remedio en la involución senil femenina? Rev Cub Obstet Ginecol 2000;26: 91-8.

8. Lama P, Leung T, Haines C, Hung T. Climacteric symptoms and knowledge about hormonal replacement therapy among Hong Kong Chinese women aged 40-60 years. Maturitas 2003;45:99-107.

9. López V, Contreras A, Toscano T, Contreras A, García F. Valoración de conocimientos sobre el climaterio en mujeres andaluzas. Aten Prim 2000;26: 476-81.

10. Rivas E, Navarro D, Damary A. Factores relacionados con la demanda de atención médica durante el climaterio. Rev Cub Endocrinol 2006;17(2):0-0. HaIlado en: http://bvs.sld.cu/revistas/end/vol17_2_06/ end04206.htm

11. Arena O. Incorporación y cumplimiento de la terapia de reemplazo hormonal del climaterio femenino antes y después del estudio WHI: revisión de la evidencia. Rev Chil Obstet Ginecol 2005;70:186-95.

12. Olazábal U, García R, Montero J, García J, Pastor F. La atención a la mujer menopáusica: un objetivo a desarrollar desde la atención primaria. Aten Prim 2000;26:405-14.

13. Solsol G, Salvador J, Maradiegue EY. Nivel de conocimiento y aceptación de la terapia de reemplazo hormonal en mujeres mayores de 39 años. Ginecol Obstet (Perú) 1997;43(3):196-200. Disponible: http:/ /sisbib.unmsm.edu.pe/Bvrevistas/ginecologia/ Vol_43N3/nivel_de_conocimiento.htm

14. Araya A, Urrutia M, Cabieces B. Climaterio y postmenopausia: aspectos educativos a considerar según la etapa del periodo. Ciencia y Enfermería 2006;12:19-27.

15. Herrera R, Llaca V. Trastornos sexuales durante el climaterio. México: Revista del Climaterio 2004; 7(38):62-77.

16. Velasco-Murillo V, Navarrete-Hernández E, OjedaMijares R, Pozos-Cavanzo J, Camacho-Rodríquez 
M, Cardona-Pérez J. Experience and knowledge about climateric and menopause in women in Mexico City. Gac Med Mex 2000;136:555-64.

17. Lugones M, Quintana T, Cruz Y. Climaterio y menopausia. Importancia de su atención en el nivel primario. Rev Cub Med Gral Integral 1997;13:494-503.

18. Pacheco J, Salvador J. Conocimiento y actitudes de la mujer peruana sobre climaterio y menopausia. Rev Per Ginecol Obstet 1998;44:157-61.

19. Navarro J, Calaf J, Comino R, Parrilla J, Magnani E, Ferrer J, Pérez R. El climaterio. Barcelona. Mediterráneo 1999;97-123.
20. Garcia F, Lopez V, Toronjo A, Toscano T, Contreras A. Evaluation of knowledge about climacteric in Andalusian women. Aten Prim 2000;26:476-81.

21. Tsao L, Chang W, Hung L, Chang S, Chou P. Perimenopausal knowledge of mid-life women in northern Taiwan. J Clin Nurs 2004;13:627-35.

22. Martin A, Del Barrio M, Del Barrio T, Fernandez $P$, Tello J. Use of health services by the climacteric women in primary health care: the need for an integral approach. Eur J Epidemiol 1999;15:231-6. 